\title{
Large optical nonlinearity and fast response time in low-temperature grown GaAs/AIAs multiple quantum wells
}

\author{
Tsuyoshi Okuno a) and Yasuaki Masumoto \\ Institute of Physics, University of Tsukuba, Tsukuba, Ibaraki 305-8571, Japan \\ Masashi Ito and Hiroshi Okamoto \\ Department of Materials Technology, Faculty of Engineering, Chiba University, Inage-ku, \\ Chiba 263-8522, Japan
}

(Received 22 February 2000; accepted for publication 15 May 2000)

\begin{abstract}
We have investigated optical nonlinearity in low-temperature (LT) molecular-beam-epitaxy-grown GaAs/AlAs multiple quantum wells (MQWs). Minimum saturation intensity, that is, maximum optical nonlinearity, is observed at around the excitonic absorption peak. The saturation intensity of the LT MQW is smaller by an order of magnitude than that of LT bulk GaAs. The response time of the LT MQW is $1 / 4-1 / 2$ of the LT GaAs, and becomes less than 1 ps, corresponding to $\sim 1 / 400$ of the standard-temperature-grown MQW. These results demonstrate a clear advantage of the room-temperature excitons in the LT MQW having large optical nonlinearity as well as fast response time. (C) 2000 American Institute of Physics. [S0003-6951(00)03627-5]
\end{abstract}

Low-temperature (LT) molecular-beam-epitaxy (MBE) grown III-V semiconductors have found many applications in ultrafast all-optical switching, ${ }^{1-3}$ femto- and picosecond laser pulse generation, ${ }^{4}$ and high-bit-rate optical communications systems. ${ }^{5,6}$ Ultrafast all-optical switching based on the absorption nonlinearity requires materials with two properties, i.e., a fast temporal response of the nonlinear absorption and large optical nonlinearity. From this purpose LT GaAs has been extensively investigated, and beryllium doping and annealing, for example, are reported very recently to improve their properties. ${ }^{7,8}$ A LT multiple quantum well (MQW) can be considered as another candidate. It is known that the standard-temperature (ST)-grown GaAs/Al(Ga)As MQW shows optical saturation intensity much smaller than that of the bulk GaAs, which means larger optical nonlinearity of the room-temperature excitons in the MQW. ${ }^{9}$ However, LT MBE growth usually brings about high crystaldefect densities, which broadens the excitonic absorption peak. Due to this difficulty, only a few papers have been published so far. They reported on LT GaAs/AlAs MQWs exhibiting 15 ps carrier lifetime, ${ }^{10}$ LT InGaAs/InAlAs MQWs demonstrating 1.5 ps time response, ${ }^{6}$ LT InGaAs/ InAlAs MQWs having 27 ps-sub-ps responses, ${ }^{11}$ and LT InGaAs/GaAs MQWs having picosecond responses. ${ }^{12} \mathrm{Nev}-$ ertheless, there has been no paper describing both the optical nonlinearity and the temporal response except Ref. 12, or describing a comparison between the MQW and the bulk film. In addition, the wavelength dependence of the nonlinearity showing excitonic enhancement was not described in the literature, although the excitonic property in the LT MQW is very important, as it is in the ST MQW. In this letter, by MBE we grow a LT GaAs/AlAs MQW which shows clear quantum-confined excitons at room temperature, and study both the optical nonlinearity and the picosecond dynamics in comparison with a ST MQW and LT bulk GaAs.

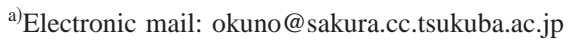

The GaAs/AlAs MQW and bulk GaAs epilayers were grown by standard MBE on semi-insulating (001) GaAs substrates. The substrates were cleaned by high-temperature treatment without As flux in a preparation chamber. ${ }^{13}$ The $\mathrm{As}_{4} / \mathrm{Ga}$ beam-equivalent pressure ratio was 5-15, and the growth rate was $1.8 \mu \mathrm{m} / \mathrm{h}$ for GaAs and $1.4 \mu \mathrm{m} / \mathrm{h}$ for AlAs. The substrate temperature, which is the most important parameter in LT MBE, was monitored and controlled by using a thermocouple placed on the substrate holder, a radiation thermometer, and the input electric power into the substrate heater. We grew first a GaAs buffer layer and then an $\mathrm{Al}_{0.3} \mathrm{Ga}_{0.7} \mathrm{As}$ etch-stop layer at $560{ }^{\circ} \mathrm{C}$. In the MQW samples, they were followed by a MQW layer consisting of 100 periods of $7 \mathrm{~nm} \mathrm{GaAs}$ wells and $7 \mathrm{~nm}$ AlAs barriers. During the MQW growth, the substrate temperature was $700{ }^{\circ} \mathrm{C}$ in ST growth and 360 or $310^{\circ} \mathrm{C}$ in the LT growth. For the bulk GaAs epilayer samples, $700 \mathrm{~nm}$ GaAs was grown instead of the MQW. A $100 \mathrm{~nm} \mathrm{Al}_{0.3} \mathrm{Ga}_{0.7}$ As window layer was grown at $450^{\circ} \mathrm{C}$. All of the epilayers were not intentionally doped. The epilayer was, then, in situ annealed at $450^{\circ} \mathrm{C}$ for $30 \mathrm{~min}$ under As pressure. An effort was made to keep the growth condition constant during the course of this study by characterizing all of the grown epilayers with x-ray diffraction, standard photoluminescence, and optical absorption measurement. Transmission electron microscope measurements showed flat heterointerfaces even in the LT MQW. For optical transmission measurements, the substrate was removed by wet-chemical etching up to the etch-stop layer. All measurements were done at room temperature.

In Fig. 1, solid lines show spectra of optical absorption $\alpha$ per unit well thickness (measured at low excitation intensity) of (a) 700, (b) 360 , and (c) $310^{\circ} \mathrm{C}$-grown MQWs. In the $700^{\circ} \mathrm{C}$-grown $\mathrm{MQW}$, sharp and well-separated quantumconfined exciton peaks [heavy-hole $(\mathrm{HH})$ and light-hole (LH) excitons] are observed. This is true also for the $360^{\circ} \mathrm{C}$ grown $\mathrm{MQW}$. For the $310^{\circ} \mathrm{C}$-grown $\mathrm{MQW}$, the excitonic peaks become obscure, but still are recognized.

For these samples, we measured optical absorption nonlinearity. As an excitation source, 2 ps pulses from a mode- 


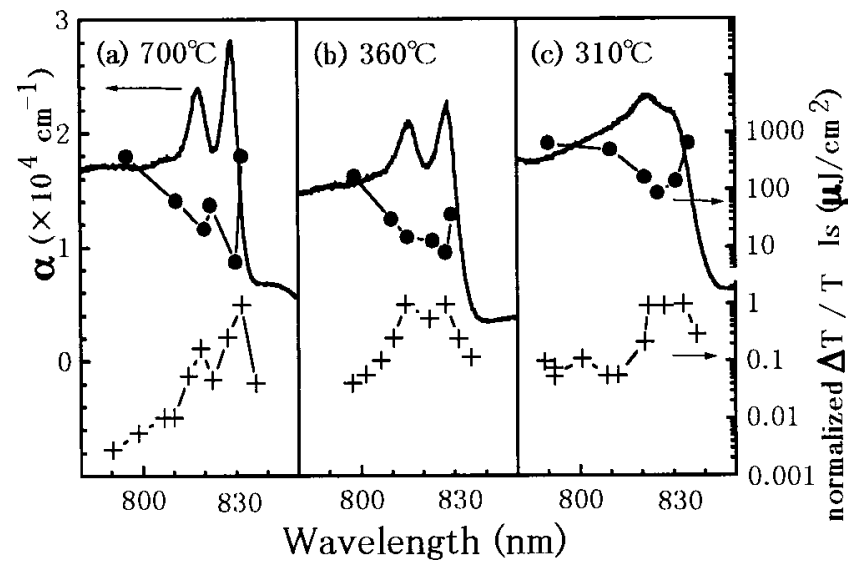

FIG. 1. Absorption spectra ( $\alpha$, solid lines), saturation densities $\left(I_{s}\right.$, circles), and the magnitude of the differential transmission at time zero in the pumpprobe measurements (normalized $\Delta T / T$, crosses) in (a) $700{ }^{\circ} \mathrm{C}$, (b) $360{ }^{\circ} \mathrm{C}$, and (c) $310^{\circ} \mathrm{C}$-grown MQWs.

locked titanium-sapphire laser were used. Incident and transmitted laser powers were measured by a calibrated $\mathrm{Si}$ photodetector. The size of the excitation spot on the sample was set to $200 \mu \mathrm{m}$ in diameter. The excitation density $I$ was changed from 0.01 to $100 \mu \mathrm{J} / \mathrm{cm}^{2}$. The maximum $I$ was limited by sample damage. The spectral width of the 2 ps laser pulse is $0.3 \mathrm{~nm}$, which is smaller by a factor of 10 than the exciton linewidth. The pulse repetition rate was set to 80 $\mathrm{kHz}$, because we found that the thermal effect changes the apparent absorption coefficient above $1 \mathrm{MHz}$ at the maximum $I$.

Figure 2(a) shows $I$ dependence of $\alpha$ measured at various wavelengths $\lambda$ for the $700{ }^{\circ} \mathrm{C}$-grown MQW. As shown in Fig. 1(a), $\lambda=830 \mathrm{~nm}$ and $\lambda=820 \mathrm{~nm}$ are HH- and LHexciton peak wavelengths and are shown by the solid circles and solid squares, respectively, in Fig. 2(a). The wavelength $\lambda=795 \mathrm{~nm}$ is located deep in the band, and $\lambda=832 \mathrm{~nm}$ is at the long-wavelength-side shoulder of the HH-exciton

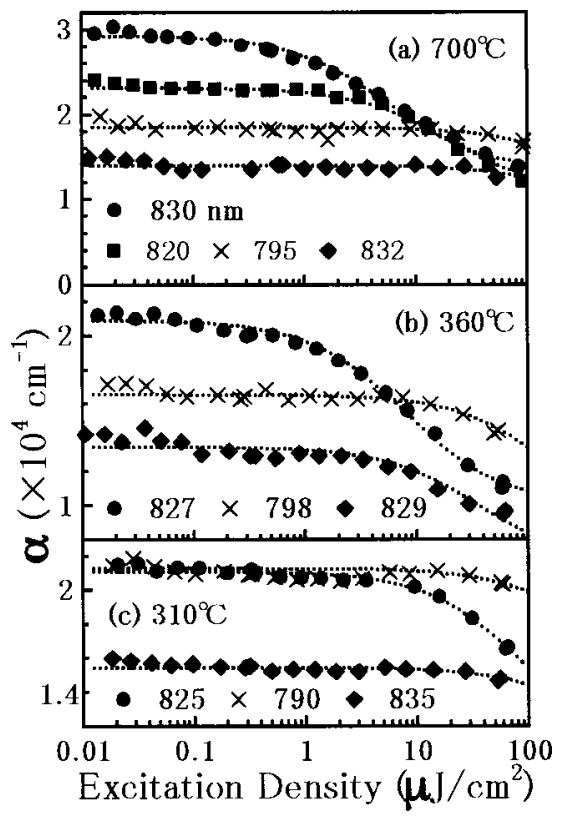

FIG. 2. Excitation density dependence of the optical absorption coefficient in (a) $700{ }^{\circ} \mathrm{C}$, (b) $360{ }^{\circ} \mathrm{C}$, and (c) $310{ }^{\circ} \mathrm{C}$-grown MQWs measured at various

wavelengths. Dashed curves are semiempirical saturation fits to the data. Sponse time $\tau$ by the $1 / e$ decay time. In the ST-grown
Downloaded 04 Feb 2004 to 130.158.105.133. Redistribution subject to AlP license or copyright, see http://apl.aip.org/apl/copyright.jsp peak. Among various $\lambda, \alpha$ begins to be reduced at the smallest $I$ at the HH-exciton peak (circles). We empirically fit these circles by an expression $\alpha=\alpha_{1} /\left(1+I / I_{s}\right)+\alpha_{2}$, where $\alpha_{1}$ and $\alpha_{2}$ are fitting parameters, and $I_{s}$ is the saturation density. ${ }^{9,14,15}$ The least-square fitting gives $1.60 /\left[1+\left(I / I_{s}\right)\right]$ $+1.32\left(\times 10^{4} \mathrm{~cm}^{-1}\right)$, where $I_{s}=5.6 \pm 0.5 \mu \mathrm{J} / \mathrm{cm}^{2}$, and is shown by the dashed line in Fig. 2(a).

In order to obtain the $\lambda$ dependence of $I_{s}$, we fitted all data obtained with various $\lambda$ by the same expression. The results are indicated by the dashed lines in Fig. 2(a), and the obtained $I_{s}$ for various $\lambda$ is plotted in Fig. 1(a) by solid circles. It is shown that $I_{s}$ becomes small at the HH- and LH-exciton peaks, and $I_{s}$ at the $\mathrm{HH}$-exciton peak is smaller than that at the LH-exciton peak. ${ }^{16}$ This shows large optical nonlinearity at the exciton peaks. ${ }^{9,14}$

Figure 2(b) shows the $I$ dependence of $\alpha$ at typical $\lambda$ for the $360{ }^{\circ} \mathrm{C}$-grown MQW. Also for this LT MQW, reduction of $\alpha$ takes place at the smallest $I$ an $\lambda=827 \mathrm{~nm}$ (HH-exciton peak) shown by solid circles. The data were fitted as previously, and the result was $1.09 /\left[1+\left(I / I_{s}\right)\right]+1.00$ $\left(\times 10^{4} \mathrm{~cm}^{-1}\right)$, where $I_{s}=8.0 \pm 0.8 \mu \mathrm{J} / \mathrm{cm}^{2} .{ }^{17}$ The saturation density $I_{s}$ is larger by $43 \%$ as compared with the $700{ }^{\circ} \mathrm{C}$ grown MQW. Other data at different $\lambda$ were fitted, and the obtained $\lambda$ dependence of $I_{s}$ is plotted in Fig. 1(b) by the solid circles. As is shown, $I_{s}$ at the LH exciton is comparable to that at the $\mathrm{HH}$ exciton.

The dependence of $\alpha$ on $I$ for the $310{ }^{\circ} \mathrm{C}$-grown MQW is shown in Fig. 2(c). In this sample, reduction of $\alpha$ occurs at the smallest $I$ at $\lambda=825 \mathrm{~nm}$ shown by the solid circles, which is located at the center of the obscured exciton peak. The least-square fitting for these circles gives $1.06 /\left[1+\left(I / I_{s}\right)\right]+1.05\left(\times 10^{4} \mathrm{~cm}^{-1}\right), \quad$ where $I_{s}=90 \pm 30$ $\mu \mathrm{J} / \mathrm{cm}^{2}{ }^{2,18}$ The saturation density $I_{s}$ is larger by more than 10 than those in the previous two samples. In Fig. 1(c), the $\lambda$ dependence of $I_{s}$ is shown by the solid circles. As shown in Figs. 1(b) and 1(c) for the LT MQW, $I_{s}$ becomes minimum at around the HH- and LH-exciton peaks, even though the exciton peak structures are broadened by LT growth. This fact shows that the optical nonlinearity becomes largest at around the excitonic absorption peak even in the LT MQW.

In order to compare the magnitude of the nonlinearity between the bulk GaAs and the MQW, we tried to measure $I_{s}$ of the bulk GaAs between $\lambda=870 \mathrm{~nm}$ (the band-gap wavelength) and $850 \mathrm{~nm}$. However, reduction in $\alpha$, such as that shown in Fig. 2, was not observed in ST or in LT bulk GaAs in the present experimental condition $\left(I<100 \mu \mathrm{J} / \mathrm{cm}^{2}\right){ }^{9,19}$ This means $I_{s}$ of the MQW is smaller by at least an order of magnitude than that of the bulk GaAs, which demonstrates the enhancement of the optical nonlinearity not only in the ST MQW (Ref. 9) but also in the LT MQW.

Temporal responses of the MQW and the bulk GaAs were measured using a degenerate pump-probe technique with 2 ps laser pulses. The pump beam energy density was $0.01 \mu \mathrm{J} / \mathrm{cm}^{2}$, and the probe density was $\sim 1 / 5$ of the pump. The wavelength $\lambda$ was set to the $\mathrm{HH}$-exciton peak for the MQW and to $865 \mathrm{~nm}$ in the bulk GaAs. Figure 3 shows differential transmission $\Delta T / T$ in the normalized scale for the MQW and the bulk GaAs as a function of the time delay between the pump and the probe beams. We define the response time $\tau$ by the $1 / e$ decay time. In the ST-grown AIP license or copyright, see http://apl.aip.org/apl/copyright.jsp 


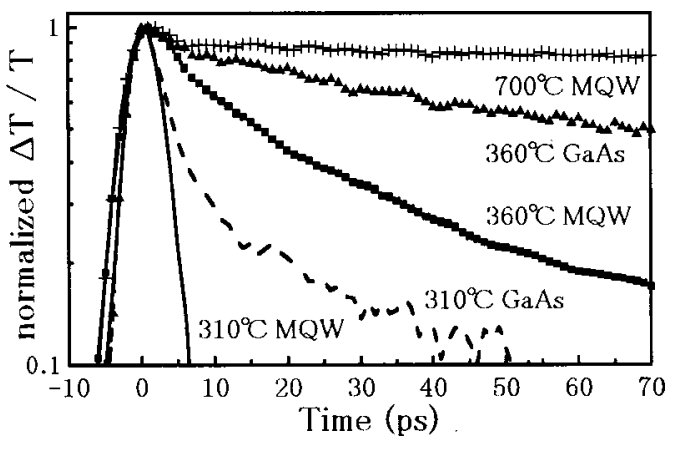

FIG. 3. Normalized temporal changes of differential transmission. The profile for the $310^{\circ} \mathrm{C}$-grown MQW shows the time resolution in this experiment.

samples, $\tau=340$ ps was obtained both for the MQW and the bulk GaAs. However, $\tau$ of the $360{ }^{\circ} \mathrm{C}$-grown MQW is decreased to $27 \mathrm{ps}$, whereas that of the $360{ }^{\circ} \mathrm{C}$-grown bulk $\mathrm{GaAs}$ is $100 \mathrm{ps}$. For the $310^{\circ} \mathrm{C}$-grown MQW, $\tau$ is faster than the time resolution. When we used 200 fs pulses for the excitation sources in place of the 2 ps pulses, $\tau$ was obtained to be 0.9 ps for this MQW. This is shorter than that of the $310^{\circ} \mathrm{C}$-grown bulk GaAs ( $\tau=2$ ps for the 200 fs excitation). These results show that the LT MQW exhibits a faster response as compared with the bulk GaAs grown at the same temperature ( $\tau$ of the LT MQW is $1 / 4-1 / 2$ of the bulk GaAs).

It is reported that positively ionized antisite As acting as electron traps is the origin of the fast response time of LT bulk GaAs. $^{7,8}$ It is also reported that in the LT GaAs/ $\mathrm{Al}(\mathrm{Ga}) \mathrm{As} \mathrm{MQW}$, the antisite As diffuses from the $\mathrm{Al}(\mathrm{Ga}) \mathrm{As}$ barrier layers to the GaAs well layers and As precipitates accumulate in the GaAs layers. ${ }^{20-22}$ Therefore, we think that in the LT MQW, the increased density of excess As in the GaAs layers or at the heterointerfaces leads to reduction in $\tau$.

The crosses in Fig. 1 show the $\lambda$ dependence of the magnitude of the differential transmission $\Delta T / T$ in the pump-probe measurements at the time zero. This value obtained at $I=0.01 \mu \mathrm{J} / \mathrm{cm}^{2}$ can be used to indicate a measure of the optical nonlinearity at various $\lambda$. As shown, the maximum in the $\lambda$ dependence of $\Delta T / T$ coincides with the minimum in the $\lambda$ dependence of $I_{s}$ in all of the three MQW samples. This result again confirms the larger optical nonlinearity around the excitonic peaks in the MQW.

We discuss the measured values of $I_{s}$. It was reported that for the standard $\mathrm{GaAs} / \mathrm{Al}_{0.3} \mathrm{Ga}_{0.7} \mathrm{As} \mathrm{MQW}$, the obtained value of $I_{S}$ corresponds to the effective exciton density of $3.3 \times 10^{17} \mathrm{~cm}^{-3}$ in the GaAs layer. ${ }^{9}$ The likely mechanism for the optical absorption nonlinearity was thought to be the filling of the excitons in the phase space density of states. ${ }^{23}$ For the $700{ }^{\circ} \mathrm{C}$-grown MQW in the present experiment, the obtained value, $I_{s}=5.6 \mu \mathrm{J} / \mathrm{cm}^{2}$, corresponds to 2.7 $\times 10^{17} \mathrm{~cm}^{-3}$, which agrees well with the above reported value. For the $310^{\circ} \mathrm{C}$-grown $\mathrm{MQW}, \tau$ is $0.9 \mathrm{ps}$, which is smaller roughly by a factor of 2 than the excitation pulse width ( $2 \mathrm{ps})$. Thus, the obtained $I_{s}\left(90 \mu \mathrm{J} / \mathrm{cm}^{2}\right)$ should be regarded as $40 \mu \mathrm{J} / \mathrm{cm}^{2}$, in order to make a fair comparison with the other samples having $\tau$ larger than $2 \mathrm{ps}$. Then, we find that $I_{s}$ becomes larger (from 5.6 and 8.0 to $40 \mu \mathrm{J} / \mathrm{cm}^{2}$ ) when the growth temperature decreases (from 700 and 360 to $310^{\circ} \mathrm{C}$ ). This increase in $I_{s}$ means the reduction of the Downloaded 04 Feb 2004 to 130.158 .105 .133 . Redistribution subje optical nonlinearity. It might be due to the degradation of MQW quality coming from the LT growth. However, this increase of $I_{s}$ by a factor of $\sim 7$ is much smaller than the reduction of $\tau$ by a factor of $\sim 400$ (from 340 and 27 to 0.9 ps). We can say that the LT MQW drastically reduces the response time while it does not reduce the magnitude of the optical nonlinearity as much.

In summary, the LT MQW shows a large optical nonlinearity (small saturation intensity and large differential transmission) at around the excitonic peak at room temperature. The saturation intensity of the MQW is almost the same, within a factor of 7 , regardless of the growth temperature. The response time of the LT MQW is $\sim 1 / 400$ of the ST MQW. In addition, the saturation intensity is smaller and the response time is shorter for the LT MQW as compared to the LT bulk GaAs. These results demonstrate the advantage of the LT MQW having large optical nonlinearity as well as fast response time.

${ }^{1}$ S. Gupta, J. F. Whitaker, and G. A. Mourou, IEEE J. Quantum Electron. 28, 2464 (1992)

${ }^{2}$ E. S. Harmon, M. R. Melloch, J. M. Woodall, D. D. Nolte, N. Otsuka, and C. L. Chang, Appl. Phys. Lett. 63, 2248 (1993).

${ }^{3}$ H. S. Loka, S. D. Benjamin, and P. W. E. Smith, IEEE J. Quantum Electron. 34, 1426 (1998)

${ }^{4}$ U. Keller, K. J. Weingarten, F. X. Kartner, D. Kopf, B. Braun, I. D. Jung, R. Fluck, C. Honninger, N. Matuschek, and J. Aus der Au, IEEE J. Sel. Top. Quantum Electron. 2, 435 (1996).

${ }^{5}$ R. Takahashi, Y. Kawamura, T. Kagawa, and H. Iwamura, Appl. Phys. Lett. 65, 1790 (1994).

${ }^{6}$ R. Takahashi, Y. Kawamura, and H. Iwamura, Appl. Phys. Lett. 68, 153 (1996).

${ }^{7}$ M. Haiml, U. Siegner, F. Morier-Genoud, U. Keller, M. Luysberg, R. C. Lutz, P. Specht, and E. R. Weber, Appl. Phys. Lett. 74, 3134 (1999).

${ }^{8}$ M. Haiml, U. Siegner, F. Morier-Genoud, U. Keller, M. Luysberg, P. Specht, and E. R. Weber, Appl. Phys. Lett. 74, 1269 (1999).

${ }^{9}$ D. A. B. Miller, D. S. Chemla, D. J. Eilenberger, P. W. Smith, A. C. Gossard, and W. T. Tsang, Appl. Phys. Lett. 41, 679 (1982) .

${ }^{10}$ I. Lahiri, D. D. Nolte, E. S. Harmon, M. R. Melloch, and J. M. Woodall, Appl. Phys. Lett. 66, 2519 (1995).

${ }^{11}$ P. W. Juodawlkis, D. T. McInturff, and S. E. Ralph, Appl. Phys. Lett. 69, 4062 (1996)

${ }^{12}$ U. Keller, Appl. Phys. B: Lasers Opt. 58, 347 (1994).

${ }^{13}$ K. Iizuka, K. Matsumaru, T. Suzuki, H. Hirose, K. Suzuki, and H. Okamoto, J. Cryst. Growth 150, 13 (1995).

${ }^{14}$ Y. Masumoto, S. Tarucha, and H. Okamoto, J. Phys. Soc. Jpn. 55, 57 (1986).

${ }^{15}$ B. Fluegel, M. Joffre, S. H. Park, R. Morgan, Y. Z. Hu, M. Lindberg, S. W. Koch, D. Hulin, A. Migus, A. Antonetti, and N. Peyghambarian, J. Cryst. Growth 101, 634 (1990).

${ }^{16}$ Some data such as $\lambda=795 \mathrm{~nm}$ in Fig. 2(a) do not show enough reduction in $\alpha$ even at the maximum $I$. Thus, we assumed to fix the ratio $r$ $=\alpha_{2} /\left(\alpha_{1}+\alpha_{2}\right)=0.45$ in all $\lambda$ (the error of $r$ was estimated to be 0.02 in the fitting for the $\lambda=830 \mathrm{~nm}$ data). We confirmed that $I_{s}$ is minimum at the exciton peaks regardless of the assumption.

${ }^{17}$ For the $360{ }^{\circ} \mathrm{C}$-grown MQW, $r=0.48 \pm 0.02$.

${ }^{18}$ For the $310{ }^{\circ} \mathrm{C}$-grown MQW, $r=0.5 \pm 0.1$. In this fitting, $r$ is assumed not to increase with the decrease of the growth temperature, because one of the origins of the residual loss $\alpha_{2}$ is neutral excess As included in LTgrown layers. The condition $r>0.4$ was assumed.

${ }^{19}$ The present largest value of $I$ not reducing $\alpha$ is consistent with the reported $I_{s}$ for bulk GaAs.

${ }^{20}$ K. Mahalingam, N. Otsuka, M. R. Melloch, and J. M. Woodall, Appl. Phys. Lett. 60, 3253 (1992)

${ }^{21}$ M. N. Chang, N. T. Yeh, C. M. Lu, K. C. Hsieh, and J.-I. Chyi, Appl. Phys. Lett. 75, 52 (1999).

${ }^{22}$ B. Lita, S. Ghaisas, R. S. Goldman, and M. R. Melloch, Appl. Phys. Lett. 75, 4082 (1999)

${ }^{23}$ S. Schmitt-Rink, D. S. Chemla, and D. A. B. Miller, Phys. Rev. B 32, 6601 (1985)

AIP license or copyright, see http://apl.aip.org/apl/copyright.jsp 